\title{
Supporting Information: Nicotine Monitoring with a Wearable Sweat Band
}

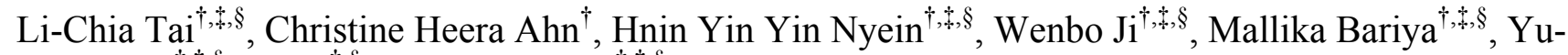

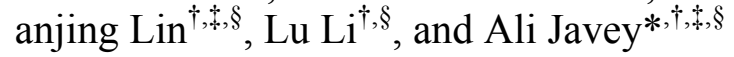

${ }^{\dagger}$ Department of Electrical Engineering and Computer Sciences, University of California, Berkeley, California 94720, United States.

${ }^{\star}$ Berkeley Sensor and Actuator Center, University of California, Berkeley, California 94720, United States.

${ }^{\S}$ Materials Science Division, Lawrence Berkeley National Laboratory, Berkeley, California 94720, United States. 


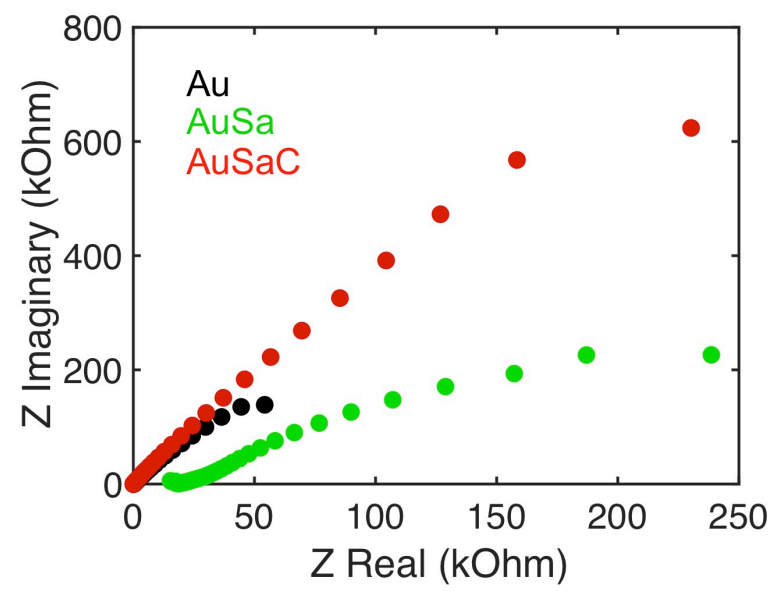

Figure S1. Electrochemical impedance spectroscopy of the gold electrode under various stages of modification. The increasing magnitude of impedance shows the addition of different modification layers onto the gold electrode. Bare gold (Au), gold and self-assembled monolayer (AuSa), gold and self-assembled monolayer and CYP2B6 enzyme (AuSaC) are shown, respectively. 


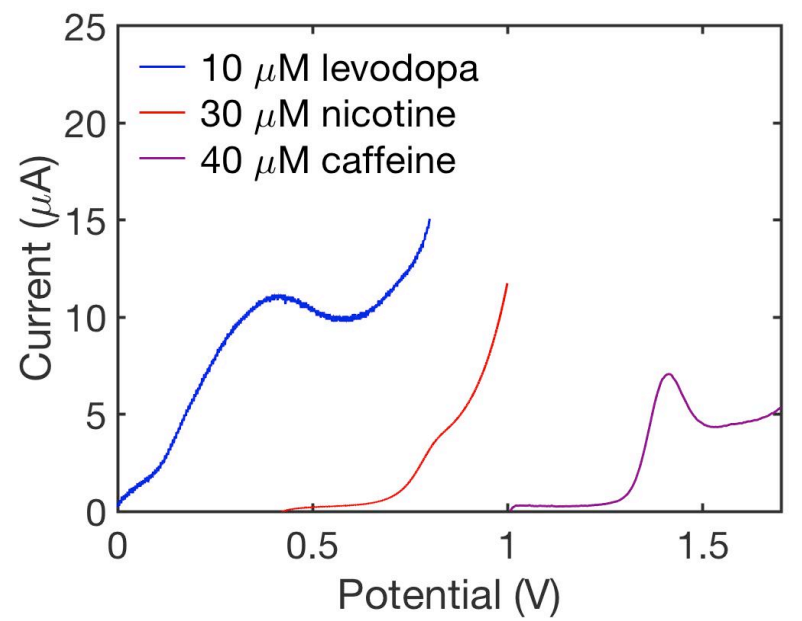

Figure S2. Oxidation peaks for selected xenobiotic molecules measured with cyclic voltammetry. The levodopa and caffeine data are from our previously published papers. ${ }^{10,13}$ 


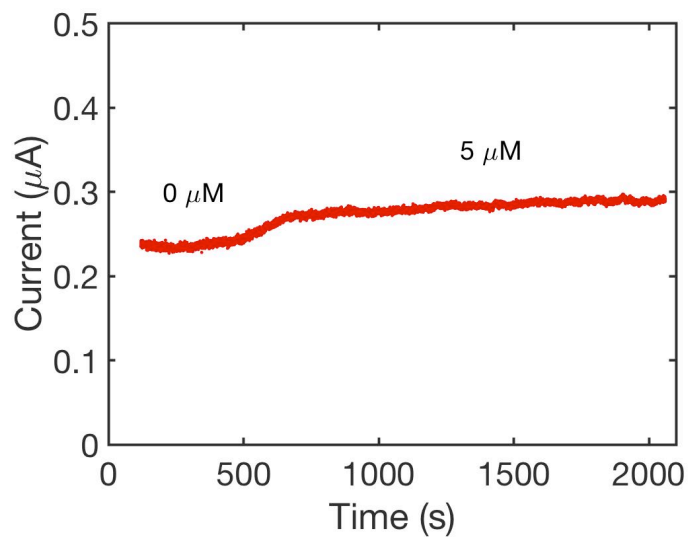

Figure S3. Long-term stability test of a functionalized working electrode. $5 \mu \mathrm{M}$ nicotine is added at 400 sec. The drift corresponds to less than $0.16 \mu \mathrm{M} / \mathrm{min}$. 